\title{
Gate dielectrics improve gallium nitride devices
}

IEEE J. Electron Dev. Soc. 8, 15-19 (2019)



Credit: W.-Z. Xu

Gallium nitride ( $\mathrm{GaN})$ heterostructures are of potential use in the development of high-power electronic devices, such as high-electron-mobility transistors (HEMTs). To achieve control over the gate leakage and channel electrostatics of GaN-based HEMTs, a gate insulator, such as hafnium oxide $\left(\mathrm{HfO}_{2}\right)$, can be introduced. However, bonds between oxygen and gallium atoms degrade the interface quality between the GaN and the $\mathrm{HfO}_{2}$, which limits device performance. The quality of the interface can be improved by inserting a high- $k$ gate dielectric between the two materials, and Wei-Zong $\mathrm{Xu}$, Hai Lu and colleagues have now shown that high- $k$ yttrium oxide $\left(\mathrm{Y}_{2} \mathrm{O}_{3}\right)$ can be used to create high-quality insertion layers because of its low oxygen permeability.
The researchers - who are based at Nanjing University - used the bilayer $\mathrm{Y}_{2} \mathrm{O}_{3} / \mathrm{HfO}_{2}$ dielectric stacks to fabricate GaN-based metal-insulator-semiconductor HEMTs on silicon. To confirm the quality of the interface between the dielectric and $\mathrm{GaN}$, measurements were carried out that showed a low interface density of states $\left(10^{12} \mathrm{~cm}^{-2} \mathrm{eV}^{-1}\right)$. The transistors exhibited a low subthreshold swing of $70 \mathrm{mV} \mathrm{dec}{ }^{-1}$ because of the small effective oxide thickness ( $2 \mathrm{~nm}$ ) of the oxide layers. Compared with transistors based on other gate dielectric materials, the bilayer dielectric stacks result in a lower gate leakage of $10^{-12} \mathrm{~A} \mathrm{~mm}^{-1}$.

Christiana Varnava

Published online: 24 January 2020 https://doi.org/10.1038/s41928-020-0371-6 